\title{
BioRePortAP, an electronic clinical record coupled with a database: an example of its use in a single centre
}

\author{
R Campanilho-Marques, , J Polido Pereira, A M Rodrigues, F Ramos, M J Saavedra, M Costa, J A Pereira da Silva, \\ H Canhão, J E Fonseca
}

From 5th European Workshop on Immune-Mediated Inflammatory Diseases

Sitges-Barcelona, Spain. 1-3 December 2010

\section{Aims}

To evaluate the efficacy and safety of the treatment of psoriatic arthritis (PsA) patients with tumor necrosis factor (TNF) antagonists in the Rheumatology Dept. of Hospital de Santa Maria using the BioRePortAP.

\section{Methods}

The Portuguese Society of Rheumatology (SPR) developed an electronic medical chart coupled with a database for the follow up of PsA patients, the BioRePortAP, which was launched in May 2009. This evaluation was based on all the PsA patients that were on active treatment with TNF antagonists in September 2009 and were registered in the BioRePortAP. All the previous data on these patients were introduced in BioRePortAP using the prospective paper based follow up protocol that this Dept. was using since 1999. Only patients with more than 9 months of treatment were analyzed.

\section{Results}

Forty-two patients with PsA, actively treated with antiTNF agents in September 2009, for at least 9 months, were analyzed in BioRePortAP. Twenty-three patients were male (55\%) and nineteen were female (45\%). The average age of these patients was $49.8 \pm 10.9$ years old, the average disease duration was of $10.7 \pm 5.6$ years and the mean duration of biological therapy was of $37.8 \pm 27.8$ months. For the $81 \%$ of patients with peripheral joint disease there was a mean reduction of more than $80 \%$ in the swollen and tender joint counts, and almost $50 \%$ in the HAQ value. In the $19 \%$ of the patients with axial involvement the reduction of BASDAI and BASFI was not statistically significant. On top of that, PASI score suffered a reduction of $64 \%$. Fourteen patients $(33.3 \%)$ had to switch their TNF antagonist treatment. $58.8 \%$ of the switches were due to adverse effects and $41.2 \%$ due to therapy failure. Regarding the 56 adverse reactions registered, only one was a severe reaction. The remaining adverse reactions were not severe and $67 \%$ of them were due to infections.

\section{Discussion}

The results of this first report of the use of the BioRePortAP in clinical practice confirm the efficacy and safety of TNF antagonist treatment in PsA. The results shown here elucidate the potential applications of BioRePortAP as a tool for efficacy and safety assessment of PsA patients treated with biotechnological drugs.

Published: 25 November 2010

doi:10.1186/1479-5876-8-S1-P63

Cite this article as: Campanilho-Marques et al:: BioRePortAP, an

electronic clinical record coupled with a database: an example of its use

in a single centre. Journal of Translational Medicine 2010 8(Suppl 1):P63. 